\title{
Inverse Analysis and Modeling for Tunneling Thrust on Shield Machine
}

\author{
Qian Zhang, ${ }^{1,2}$ Yilan Kang, ${ }^{1}$ Zheng Zheng, ${ }^{1}$ and Lihui Wang ${ }^{3}$ \\ ${ }^{1}$ Key Laboratory of Modern Engineering Mechanics, Tianjin University, Tianjin 300072, China \\ ${ }^{2}$ State Key Laboratory of Shield Machine and Boring Technology, China Railway Tunnel Group Co., Ltd, Zhengzhou 450001, China \\ ${ }^{3}$ Department of Military Vehicle, Academy of Military Transportation, Tianjin 300161, China
}

Correspondence should be addressed to Yilan Kang; tju_ylkang@tju.edu.cn

Received 7 October 2013; Accepted 1 November 2013

Academic Editor: Cheng Shao

Copyright (C) 2013 Qian Zhang et al. This is an open access article distributed under the Creative Commons Attribution License, which permits unrestricted use, distribution, and reproduction in any medium, provided the original work is properly cited.

With the rapid development of sensor and detection technologies, measured data analysis plays an increasingly important role in the design and control of heavy engineering equipment. The paper proposed a method for inverse analysis and modeling based on mass on-site measured data, in which dimensional analysis and data mining techniques were combined. The method was applied to the modeling of the tunneling thrust on shield machines and an explicit expression for thrust prediction was established. Combined with on-site data from a tunneling project in China, the inverse identification of model coefficients was carried out using the multiple regression method. The model residual was analyzed by statistical methods. By comparing the on-site data and the model predicted results in the other two projects with different tunneling conditions, the feasibility of the model was discussed. The work may provide a scientific basis for the rational design and control of shield tunneling machines and also a new way for mass on-site data analysis of complex engineering systems with nonlinear, multivariable, time-varying characteristics.

\section{Introduction}

With the rapid development of sensor and detection technologies, the on-site measurement of large engineering equipment can now provide extensive and real-time mass measured data, which include the evolution information of the key control variables during equipment operation and other main influencing factors. Determining the internal relation among these main influencing factors in miscellaneous and noisy data and building an evolution model that can represent the inherent law are significant to the design, control, and safe operation of complex engineering equipment.

Data mining technology has provided technological tools for the analysis and modeling of mass engineering data and has been effectively applied in measured data analyses in various fields, such as product and process design, equipment control, and fault detection [1]. The common data mining tools used in engineering data analysis at present include statistical (regression analysis, correlation analysis, cluster analysis, etc.) and artificial intelligence methods (fuzzy theory, rough set, neural network, genetic algorithm, etc.). The regression analysis method, which is characterized by high error-tolerant ability, transparent mining course, and high controllability, has an important function in the modeling and mining of the law between variables in continuous mass engineering data [2]. Moreover, some new methods in artificial intelligence, pattern recognition, and machine learning have been introduced in many engineering data analyses [3-5].

Shield tunneling machine, a kind of typical equipment utilized in underground engineering, is characterized by a complex structure and a heavy-duty mechanism. This equipment has a significantly high requirement for safe operation and control because it operates in complex geological conditions and extreme multifield underground environments. Tunneling thrust is the most important control variable in a shield tunneling machine which reflects the geological adaptability of the equipment. The thrust value can directly affect the tunneling status and efficiency of the equipment. 
The composition of the total thrust is very complex and usually includes dozens of components which are closely related to the geological condition, the equipment structure, and operating status. However, the influence laws between the thrust and those factors remain unclear. Therefore, analyzing the effective information in mass tunneling data recorded by the equipment airborne system and studying the correlation among the major control parameters are important for the setting and control of the tunneling thrust and for ensuring safe and efficient construction.

The classic Krause empirical formula is currently the main formula adopted to estimate the shield tunneling thrust [6]. Krause, a German engineer, proposed an empirical formula $F=\beta D^{2}$ ( $D$ is the diameter and $\beta$ is the empirical coefficient) through the statistical analysis on engineering data from hundreds of shield machines [7]. This empirical formula describes the power relation between the thrust and the equipment diameter. However, all the other factors, such as construction geological conditions and machine operating status, are included in the wide-range empirical coefficient $\beta$, as shown in Figure 1. In the early stage of thrust design, $600 \mathrm{kN} / \mathrm{m}^{2}$ was used as the experience thrust value on unit area of the tunneling interface [8]. Besides, Zhang et al. [9] developed a linear regression model based on experimental data analysis which took the thrust, the cutterhead rotating speed, and the chamber pressure as independent variables and the advance rate as the dependent variable. Ates et al. [10] discussed the statistical relationships between TBM diameter and installed thrust capacity, nominal cutterhead torque capacity, total weight, maximum rotational speed of cutterhead, and number of disc cutters based on a database including 262 TBMs' design parameters. Hassanpour et al. [11] proposed a performance prediction model which considered two rock characteristic parameters as independent variables through regression data analysis. Yagiz et al. [12] carried out a regression model of the advance rate considering five geological parameters, in which four independent variables have a linear relationship with the advance rate except one. Grima et al. [13] presented a similar model of the advance rate using the neural-fuzzy method. Zhang et al. [14] proposed a regression model of the energy consumption combined with mechanical analysis. Gong and Zhao [15] analyzed the on-site data from a project in Singapore to discuss the relation between the rock brittleness and the tunneling performance. Hamidi et al. [16] discussed the mapping relation between rock properties and the advance rate through Rock Mass Rating sorting technique. Benardos and Kaliampakos [17] established a neural network model of the penetration considering rock property parameters, the thrust force of single cutter, the cutter dimension, and the rotating speed. Zhou et al. [18] proposed a prediction model for the air chamber pressure control in slurry shield tunneling through the PSO-based Elman neural network method.

Data mining technology has been gradually applied in the analysis of mass measured data to extract the important relation among the parameters contained in shield engineering. However, many problems remain in the actual use of this technology. First, most of the regression models built

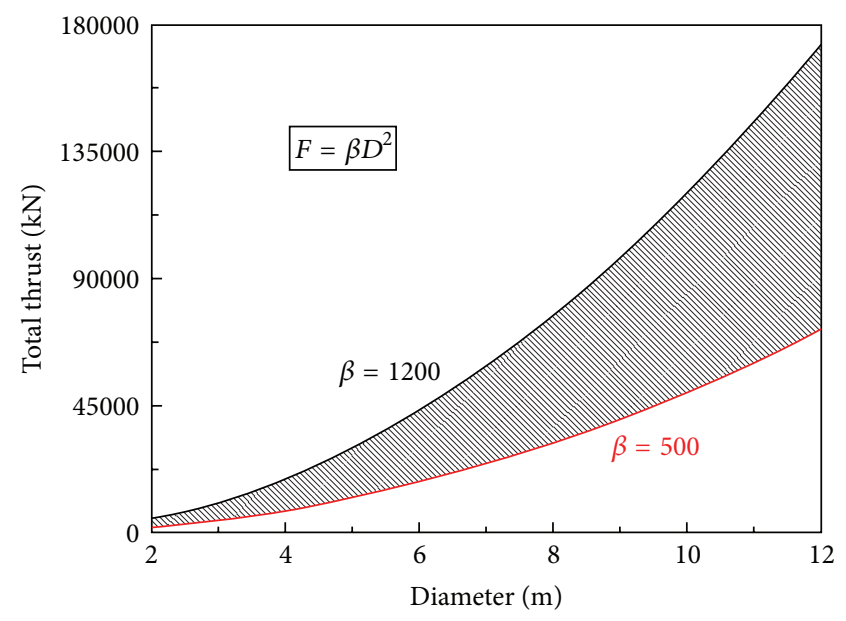

FIGURE 1: Predicted thrust range by Krause empirical formula.

for the above mentioned work are linear or quasilinear regression models, which exhibit difficulty in recognizing the nonlinear structure as a explicit model. Recognizing and building a explicit function expression with a definite physical connotation and control law from the seemingly "black box" mass data are the difficult points. Second, existing research lacks the internal-mechanism-analysis-based theoretical direction in the selection and representation of engineering parameters and cannot effectively describe the internal relation among core factors. Lastly, no instructive modeling standard exists for the equal treatment and direct adding of independent variables in different classes and dimensions during the modeling of multivariable problems. These problems are difficult to be solved only relying on the development of mining technologies. The nature and characteristics of specific project objects for research must be combined. With the development of data inverse analysis research, problem-based internal mechanism analysis and data mining technology need to be combined to facilitate the application of the measured data analyses in complex engineering system modeling.

This paper aims to conduct a systematical analysis of mass on-site data from the airborne recording system of shield machines in order to identify and build an explicit function expression for tunneling thrust. Dimensional analysis method is integrated with the regression and residual analysis technologies used in modeling. A systematic mass on-site data inverse analysis method is then established. The influencing factors of the thrust are detailed analyzed. A mechanical model of tunneling thrust is built, and the effectiveness of the model is discussed with on-site data from several project cases.

\section{Mechanical Characteristic Analysis and Modeling of Tunneling Thrust}

A shield machine, which is usually composed of several parts, can perform integrated-underground construction, including excavation, deslagging, and lining. Figure 2 gives 


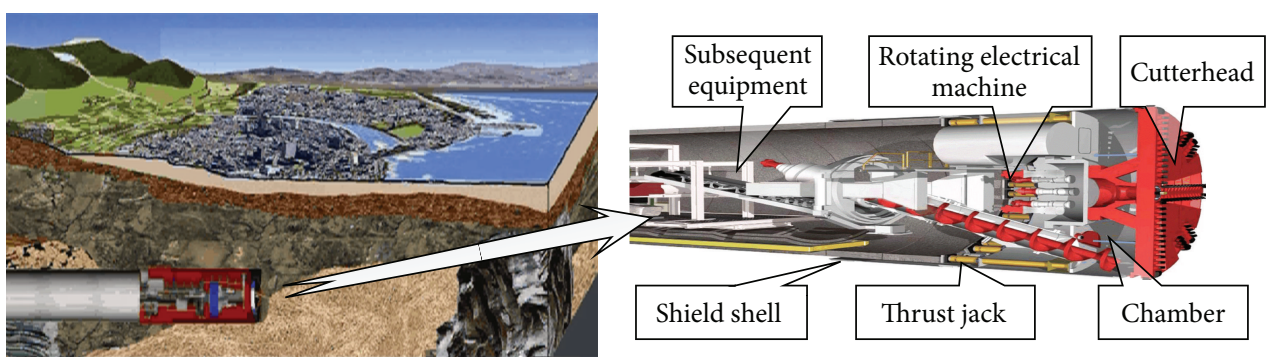

FIGURE 2: Schematic of a shield tunneling machine.

a schematic description of a shield machine's complex structure. Each part of the equipment generates constant complex mechanical effects on the surrounding ground, which contributes to the tunneling thrust. Therefore, the total thrust can include up to more than a dozen components and change in real time with several geological and control parameters, which present difficulties in the analysis and modeling.

To analyze the main factors influencing the tunneling thrust, they can be described by three kinds of parameters including operating parameters, geological parameter, and structural parameter as shown in Figure 3. In the tunneling process of a shield machine, some thrust components are relatively stable and are not influenced by tunneling speed, such as the earth pressure at rest, the groundwater pressure, the shield shell friction, and subsequent equipment traction. Some others are directly related to tunneling speed, such as the thrust acting on the cutterhead which will increase when the machine tunneling fast. Considering these characteristics of the tunneling thrust, the paper divides it into the external thrust and the operating thrust, which can be expressed as

$$
\begin{gathered}
F=F_{o}+F_{e}, \\
F_{o}=f(W, v, \omega), \\
F_{e}=g\left(W, D, H, H^{\prime}\right),
\end{gathered}
$$

where $F$ is the total tunneling thrust; $F_{o}$ is the operating thrust which is mainly influenced by the machine advance rate $v$, the cutterhead rotating speed $\omega$, and the ground bearing capacity $W ; F_{e}$ is the external thrust which is mainly influenced by the shield diameter $D$, the ground bearing capacity $W$, the depth of overburden $H$, and the groundwater level $H^{\prime} ; f()$ and $g()$ represent the functional relation among parameters. Modeling for the tunneling thrust is to determine the specific function expressions of $f()$ and $g()$.

The advance rate of the machine $v(\mathrm{~m} / \mathrm{min})$ and the rotating speed of cutterhead $\omega(\mathrm{rev} / \mathrm{min})$ are the core parameters to describe the operating status of the machine. The paper introduces the tunneling penetration $\delta(\mathrm{m} / \mathrm{rev})$, which is the cutting depth that the cutterhead completes in every circle of rotation. The value of the tunneling penetration equals the ratio of the advance rate and the rotating speed:

$$
\delta=\frac{v}{\omega} \text {. }
$$

The tunneling penetration reflects the influence of both the advance rate and the cutterhead rotating speed. Therefore,

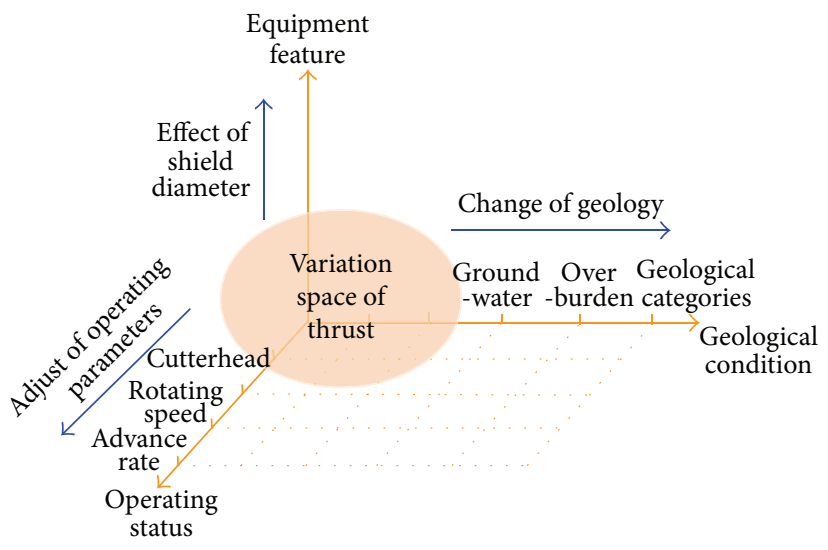

FIGURE 3: Analysis on main factors influencing tunneling thrust.

it may be used as the comprehensive characteristic index of the operating status. Equation (2) can be simplified as

$$
F_{o}=f(W, \delta) \text {. }
$$

Considering that the chamber support pressure is related to earth pressure at rest and groundwater pressure and the acting pressure is basically in the balance status on tunneling interface, the chamber support pressure $P(\mathrm{kPa})$ may be used as an approximate substitute for the influence of both the earth pressure at rest and the groundwater pressure. Equation (3) can be simplified as

$$
F_{e}=g(W, D, P) .
$$

Basic physical laws and the dimensional analysis method are adopted in this study to determine the specific function expressions of $f()$ and $g()$. Dimensional analysis method reveals the physical essence of the problem through the dimensional attribute analysis of parameters, thereby providing an effective tool for the research on complex engineering problems. According to the dimensional analysis theory, only the physical variables with the same dimension can be added. Then the specific functional relation of $f()$ and $g()$ can be determined based on dimensional analysis combined with the mechanical analysis for the shield tunneling process:

$$
\begin{gathered}
F_{o} \propto W^{a} \delta^{b}, \\
F_{e} \propto P^{c} D^{e}+W^{d} D^{e} .
\end{gathered}
$$


Then the thrust model can be expressed as

$$
F=F_{o}+F_{s}=\beta_{1} W^{a} \delta^{b}+\left(\beta_{2} P^{c}+\beta_{3} W^{d}\right) D^{e}
$$

where $\beta_{1}, \beta_{2}$, and $\beta_{3}$ are the undetermined coefficients, whose values are related to the specific project condition and can be identified by on-site data analysis; $a, b, c, d$, and $e$ are the undetermined exponents, which can be determined through dimensional equilibrium principle.

The functional relation among variables in (8) needs to satisfy the principle of dimension consistency. The dimensional expression of tunneling thrust is $[M][L][T]^{-2}$. The physical variables in the operating thrust $F_{o}$ and the external thrust $F_{s}$ can be expressed using three basic dimensions, namely, length $[L]$, mass $[M]$, and time $[T]$. The dimension of the ground bearing capacity $W$ and the chamber support pressure $P$ is $[M][L]^{-1}[T]^{-2}$, and that of the penetration $\delta$ and the diameter $D$ is $[L]$. Equation (8) can be expressed in dimensional form as follows:

$$
\begin{aligned}
{[M][L][T]^{-2}=} & {[M]^{a}[L]^{-a}[T]^{-2 a}[L]^{b} } \\
{[M][L][T]^{-2}=} & {[M]^{c}[L]^{-c}[T]^{-2 c}[L]^{e} } \\
& +[M]^{d}[L]^{-d}[T]^{-2 d}[L]^{e}
\end{aligned}
$$

The dimension consistency principle requires that the dimensions on the two sides of (9) be equal; thus, the undetermined exponents are $a=c=d=1, b=2$, and $e=2$. The general formula of the tunneling thrust with dimensionless undetermined coefficients $\beta_{1}, \beta_{2}$, and $\beta_{3}$ are then obtained as

$$
F=\beta_{1} W \delta^{2}+\left(\beta_{2} W+\beta_{3} P\right) D^{2}
$$

Equation (10) gives the basic architecture of the shield tunneling thrust model, in which the relation between the main factors, including the operating status, the geological conditions, and the equipment structure, and the tunneling thrust is presented. If the geological parameter $W$ is small, the required tunneling thrust is also small. Under similar geological conditions, the increase in the tunneling thrust is related to the square of the penetration when tunneling fast. If the equipment tunnels with a large overburden, the total thrust will also increase. In (10), the tunneling thrust has a square relation with the equipment diameter, which is consistent with the balance relation in mechanics and the thrust-diameter relation provided in the Krause empirical formula.

\section{Inverse Identification of Shield Tunneling Thrust Based on Mass On-Site Data}

The inversion identification of the dimensionless undetermined coefficients in the general formula (10) can be conducted based on specific engineering data and with the multiple regression method. The multiple regression method, one of the basic methods in data mining, can be used to determine the explicit function relation of the two groups of
TABLE 1: Basic statistical characteristics for the data.

\begin{tabular}{lcccc}
\hline & Minimum & Maximum & Average & $\begin{array}{r}\text { Standard } \\
\text { deviation }\end{array}$ \\
\hline $\begin{array}{l}\text { Thrust }(\mathrm{kN}) \\
\begin{array}{l}\text { Advance rate } \\
\text { (mm/min) }\end{array}\end{array}$ & 10436.07 & 17440.65 & 13376.89 & 1321.27 \\
$\begin{array}{l}\text { Rotating speed } \\
\text { (rev/min) }\end{array}$ & 0.90 & 1.10 & 1.02 & 0.07 \\
$\begin{array}{l}\text { Penetration } \\
\text { (mm/rev) }\end{array}$ & 22.76 & 45.89 & 35.91 & 5.89 \\
$\begin{array}{l}\text { Pressure in } \\
\text { chamber (MPa) }\end{array}$ & 0.19 & 0.30 & 0.23 & 0.017 \\
$\begin{array}{l}\text { Ground bearing } \\
\text { capacity (kPa) }\end{array}$ & 72.83 & 90.46 & 83.37 & 5.97 \\
\hline
\end{tabular}

variables. In the method, the target variables are dependent and the physical variables that cause constant changes in the dependent variables are independent. The analysis aims to determine the quantitative relation between the dependent and independent variables through constant iterative computation and result judgment.

The project used in the regression analysis is the 700-ring construction area of the Tianjin Metro Line 9 from Shiyijing Lu Station to Dazhigu Xilu Station in China. Komatsu Ltd. is selected to provide TM634PMX earth pressure balance shield with a diameter of $6.34 \mathrm{~m}$. The tunnel has an overburden of about $8-11 \mathrm{~m}$. The main geological strata concerned include mucky soil, silty clay, silt, silty sand, and so forth. The geological parameters involved in the analysis were adopted according to the actual situation of engineering, which was provided through in situ geotechnique tests. The operating and control parameters used were recorded in real time by the machine during working. The on-site data out of 350 rings of this project is used as training sample, and the data of the other 250 rings will be reserved as testing sample. The basic statistical characteristics of the data is given in Table 1.

Based on the least square method, the professional statistical analysis software SPSS (Statistical Product and Service Solutions) is used to complete the regression computation. The optimal values of the undetermined coefficients are then determined, and the specific expression of the tunneling thrust is obtained as (11). The significance of the regression computation is preliminarily verified through the combination of $F$ - and $t$-tests:

$$
F=1.8 \times 10^{4} W \delta^{2}+(1.2 W+0.79 P) D^{2} .
$$

The statistical testing results of the regression computation are shown in Table 2. The linear correlation coefficient reflects the level of linear correlation of the variables, which is between 0 and 1. A large value of the coefficient indicates obvious linear correlation. This coefficient can be used to discuss the rationality of the regression model structure. $F$ test is adopted to analyze the overall regression effect, and $t$-test is employed to determine the significance of every regression coefficient. The closer the computed result of the associated probability (the value of Prob. in Table 2) is to 
TABLE 2: Statistical testing results of the regression computation.

(a) Linear correlation coefficient

\begin{tabular}{lccc}
\hline Compositional independent variable & $P D^{2}$ & $W D^{2}$ & $W \delta^{2}$ \\
\hline Correlation coefficient & 0.996 & 0.992 & 0.967 \\
Prob. & 0.000 & 0.000 & 0.000 \\
\hline
\end{tabular}

(b) F-test

\begin{tabular}{cccc}
\hline Model $F$-test & $R^{2}$ & $F$ & Prob. \\
\hline & 0.994 & 17310.787 & 0.000 \\
\hline
\end{tabular}

(c) $t$-test

\begin{tabular}{lccc}
\hline Model $t$-test & Regression coefficient & $t$ & Prob. \\
\hline$\beta_{1}$ & 18383.878 & 9.800 & 0.000 \\
$\beta_{2}$ & 1.179 & 7.877 & 0.000 \\
$\beta_{3}$ & 0.792 & 13.268 & 0.000 \\
\hline
\end{tabular}

0 , the better regression effect it indicates. According to the results in Table 2 , the dependent variable $F$ has a close linear correlation with $W \delta^{2}, W D^{2}$, and $P D^{2}$, indicating that the nonlinear structure of the regression model is rational. The $F$ - and $t$-tests also show that the regression effect reached significant levels.

The above analysis shows that the tunneling thrust model built based on mechanical characteristic analysis and dimensional analysis methods reflects the influence of major factors and the internal relation among different parameters. The model can also provide a description of the change of tunneling thrust in relation to the geological conditions and operating status. A set of curves that describe the change between tunneling thrust under different geological conditions and tunneling penetration is illustrated in Figure 4 based on (11).

\section{Residual Analysis and Verification of the Shield Tunneling Thrust Model}

The measured value of the tunneling thrust and that predicted by the model are quantitatively compared. The difference in the two is defined as the model residual as follows:

$$
e_{n}=F_{\text {measured }}-F_{\text {predicted }}
$$

where $e_{n}$ is the model residual, which is related to the tunneling ring number. Figure 5 presents the curves of the on-site measured values and the predicted values by (11) of the shield tunneling thrust changing with the number of rings. The measured values and the predicted values are consistent in terms of change trend. Moreover, Figure 5 shows that the residual curve basically fluctuates around 0 , indicating that the model has included the main parts and the core influencing factors of the tunneling thrust. Besides, the fluctuation of the residual curve shows a little change trend, which shows that some of the secondary influencing factors of the thrust may still be in the model residual. The stationarity and randomness of the model residual data series are further

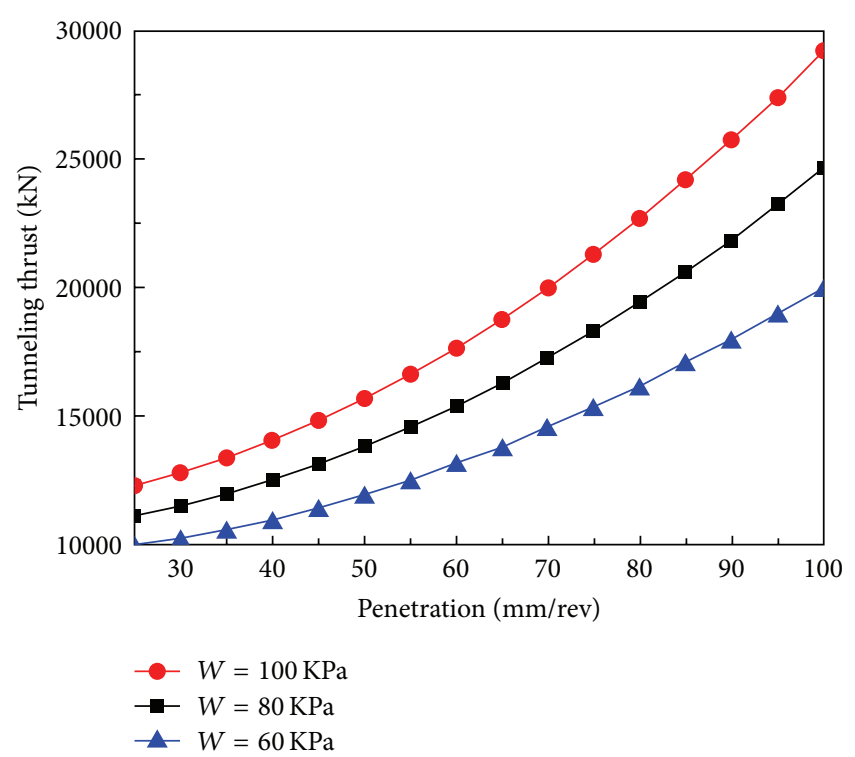

FIgURE 4: Curves of the thrust changing with the penetration under different geological conditions.

TABLE 3: Stationarity analysis of the model residual.

\begin{tabular}{lll}
\hline Phillips-Perron test statistic & PP-Stat. & Prob. \\
\hline & -13.947 & 0.000 \\
\hline
\end{tabular}

analyzed to discuss the applicability of the shield tunneling thrust model.

The PP statistical magnitude [19] is adopted to analyze the stationarity of the model residual. This statistical magnitude can describe the overall fluctuation of the residual data with the change in the ring number. Assuming that the residual data are a nonstationary series, the result (the value of Prob. in Table 3) shows that the associated probability of accepting the null hypothesis is approximately 0 . Therefore, the calculated residual of the model is a stationary series, which indicates that the model has included the major influencing factors of the tunneling thrust. The $Q$ statistical magnitude [20] is adopted to analyze the randomness of the model residual. This statistical magnitude can describe the relevance of the residual data. If the magnitude has a relatively large value, a law must be followed in the residual data. Assuming that no tunneling thrust influencing factor exists in the residual data; that is, the residual data are a pure random series, the results (the values of Prob. in Table 4) show that the associated probability of accepting the null hypothesis is approximately 0 . This finding means that the residual data of (11) does not totally randomly fluctuate, but has some secondary factors existing in it.

The residual analysis above indicates that the model (11) reflects the influence of the main parts and the core parameters of the tunneling thrust. In addition, some secondary factors and errors still exist in the model residual. The possible reasons may include the following. First, the geological data adopted in the analysis is from geological borehole measurement. Considering that the data obtained from a limited 


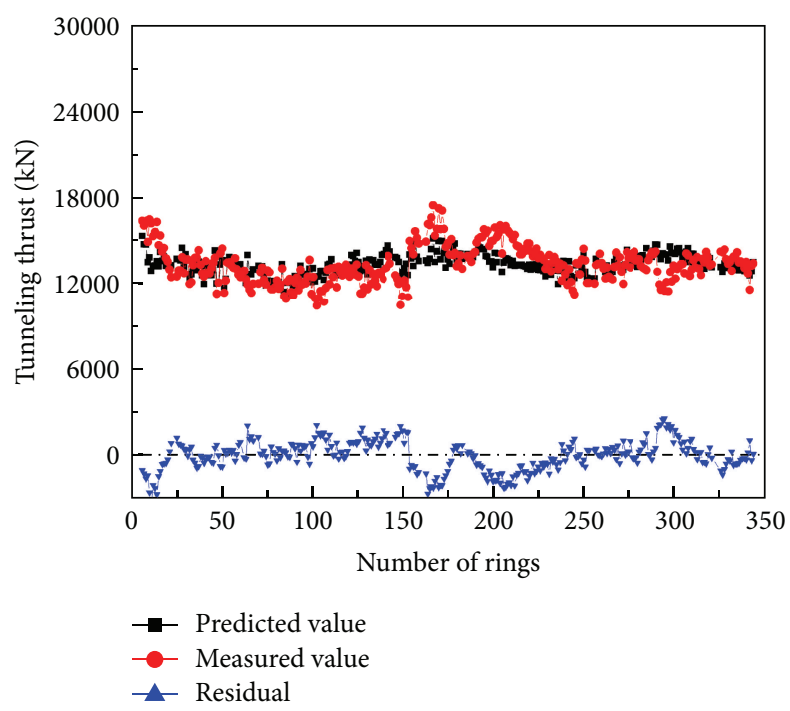

(a)

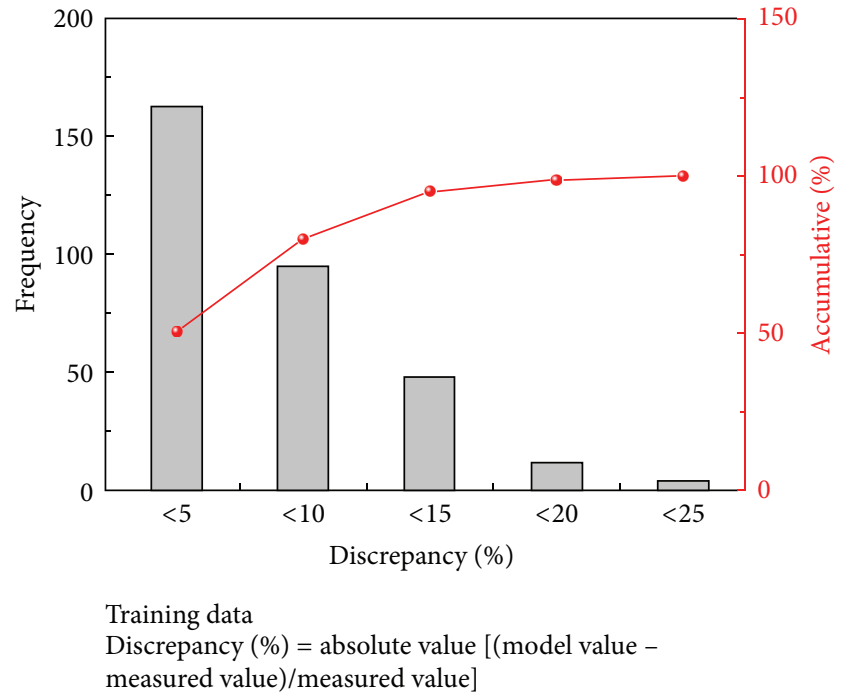

(b)

Figure 5: Residual analysis of the tunneling thrust model. (a) Comparison between the predicted thrust and the measured value. (b) Statistical histogram of the residuals.

TABLE 4: Randomness analysis of the model residual.

\begin{tabular}{lcc}
\hline Step & Q-stat. & Prob. \\
\hline 1 & 52.737 & 0.000 \\
2 & 88.529 & 0.000 \\
3 & 136.90 & 0.000 \\
4 & 160.48 & 0.000 \\
5 & 182.60 & 0.000 \\
6 & 189.86 & 0.000 \\
7 & 196.65 & 0.000 \\
8 & 204.11 & 0.000 \\
9 & 206.90 & 0.000 \\
10 & 210.77 & 0.000 \\
\hline
\end{tabular}

number of geological boreholes cannot accurately reflect the geological information of all the tunneling sections, an error may occur in the predicted results when the geological data is used in the model. Second, the effects of key geological parameters are considered in the model. However, given that the parameters considered are limited, an error may also occur in the predicted value. Third, noise exists in some onsite measured data.

The 250-ring measured data that were not involved in the modeling process are reserved as a testing sample for the model verification. This group of independent data is substituted in (11) and the predicted result of testing sample is obtained. Comparing the predicted value and the on-site measured value of the tunneling thrust, Figure 6 shows that they are basically accordant, reflecting the rationality and effectiveness of the inversion analysis and modeling method based on mass measured data proposed in the paper. The flow chart of the method is shown in Figure 7.

\section{Engineering Applications of the Shield Tunneling Thrust Model}

The tunneling thrust model built based on mechanical and dimensional analyses reflects the essential characteristics of the equipment during construction as well as the internal influence law among the core control parameters. This model can be applied in the prediction and control of the tunneling thrust in shield engineering under different engineer conditions. Equation (10) provides the general formula of the model, which should be subjected to specific projects in its application. The engineering applications of the model under two typical geological conditions, namely, sandy pebble geology and rock-soil mixed geology, were illustrated in the paper to discuss and verify the effectiveness of the model.

The first project discussed is 700-ring section between Fengtai Dongjie Station to Fengtai Beilu Station of Beijing Metro Line 9 in China. An EPB shield tunneling machine manufactured by JTSC Company in Japan was used with a diameter of $6.14 \mathrm{~m}$. The tunnel has an overburden of about $8-11 \mathrm{~m}$. The construction area studied mainly runs through sandy pebble. The range of the advance rate is 12$82 \mathrm{~mm} / \mathrm{min}$ and that of the rotating speed of cutterhead is $0.8-1.0 \mathrm{rev} / \mathrm{min}$. The penetration is changed from 14 to $92 \mathrm{~mm} / \mathrm{rev}$. The pressure inside chamber is adjusted in the range of $40-130 \mathrm{kPa}$.

The other project discussed is 400-ring section between Xiang Mihu North Station to Xiang Mei North Station of Shenzhen Metro Line 2 in China. An EPB shield tunneling machine manufactured by NFM Company in France was used with a diameter of $6.28 \mathrm{~m}$. The tunnel has an overburden of about 9-12 m. The construction area studied mainly runs through plain fill, hard plastic/moldable gravelly sand, silty clay, completely weathered granite, and strongly weathered granite. The advance rate varies in the range 


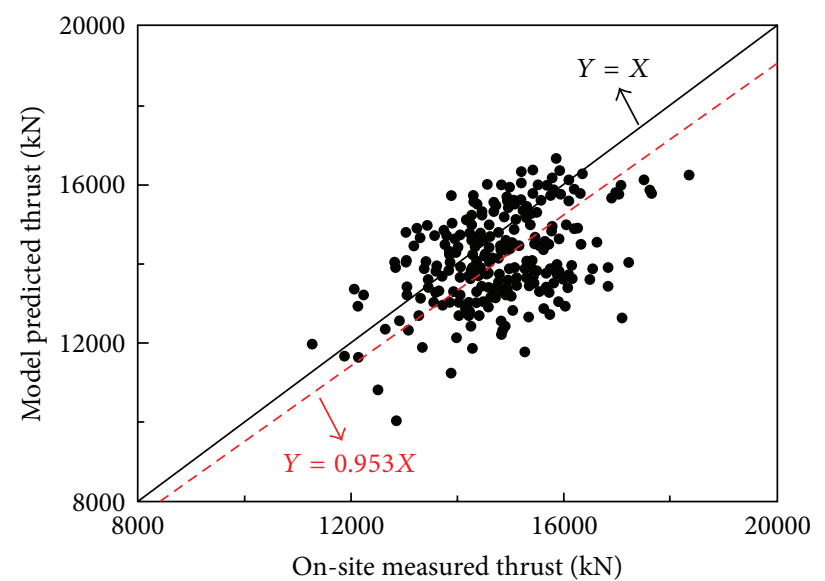

- Testing data

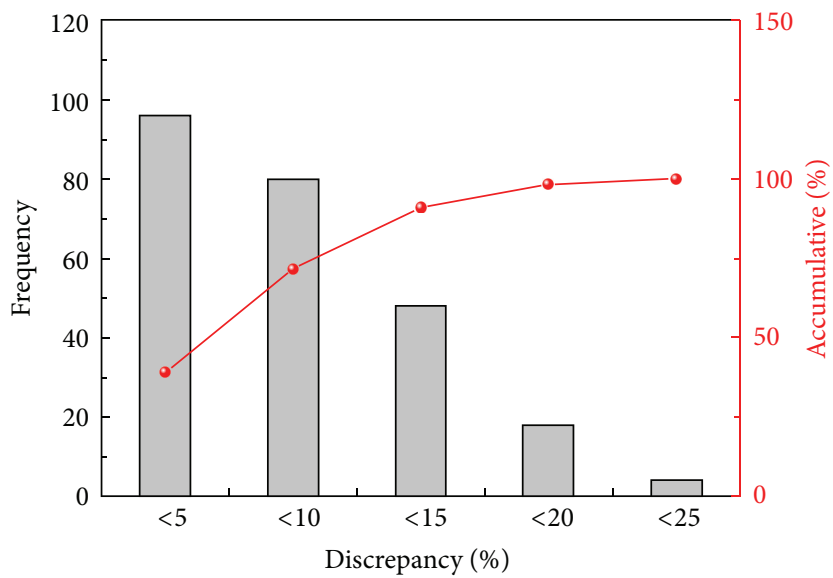

Testing data Discrepancy $(\%)=$ absolute value $[($ model value measured value)/measured value]

(b)

FiguRE 6: Independent data test of the model. (a) Cross-correlation graph of the testing data. (b) Statistical histogram of the prediction errors.

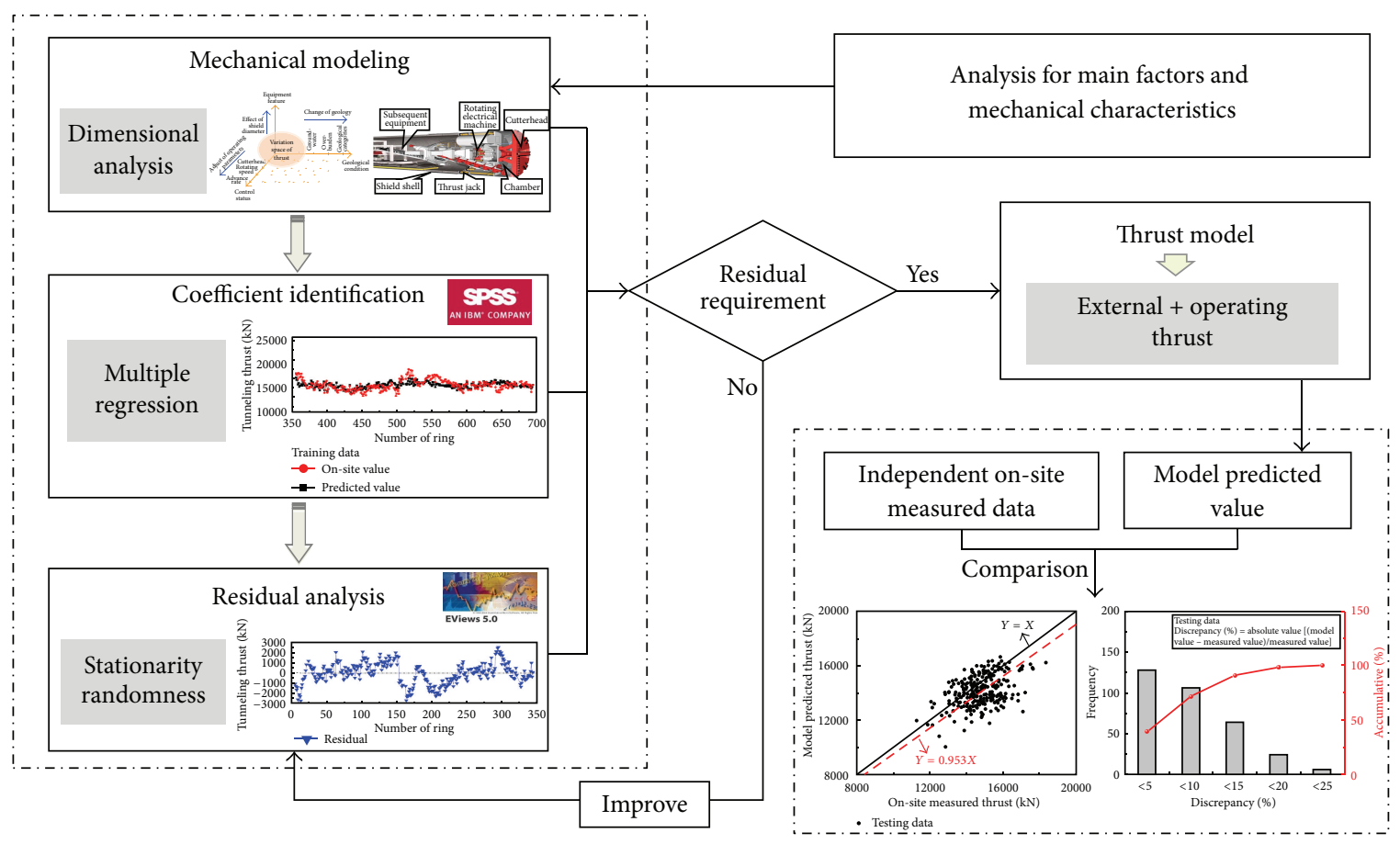

FIGURE 7: Flow chart for the mechanical analysis and modeling method based on mass on-site data.

of $12-60 \mathrm{~mm} / \mathrm{min}$. The rotating speed of the cutterhead is $1.0-1.4 \mathrm{rev} / \mathrm{min}$. The excavating depth per revolution is $11-$ $54 \mathrm{~mm} / \mathrm{rev}$. The pressure inside chamber is $113-220 \mathrm{kPa}$.

The first 100-ring data of the two projects is used to determine the coefficients in the general formula (10), from which the specific function expression of the two projects above can be obtained as shown in Table 5 . The predicted value and measured value of the tunneling thrust in the two projects are quantitatively compared and the predicted errors are statistically analyzed as shown in Figure 8 . The predicted errors of $80 \%$ data points in both of the two projects are less than $30 \%$. As the proposed model effectively extracts the core characteristics of the thrust variation, the predicted value can be consistent with the measured value, thereby providing an important reference for the prediction and control of the tunneling thrust on shield machines. 


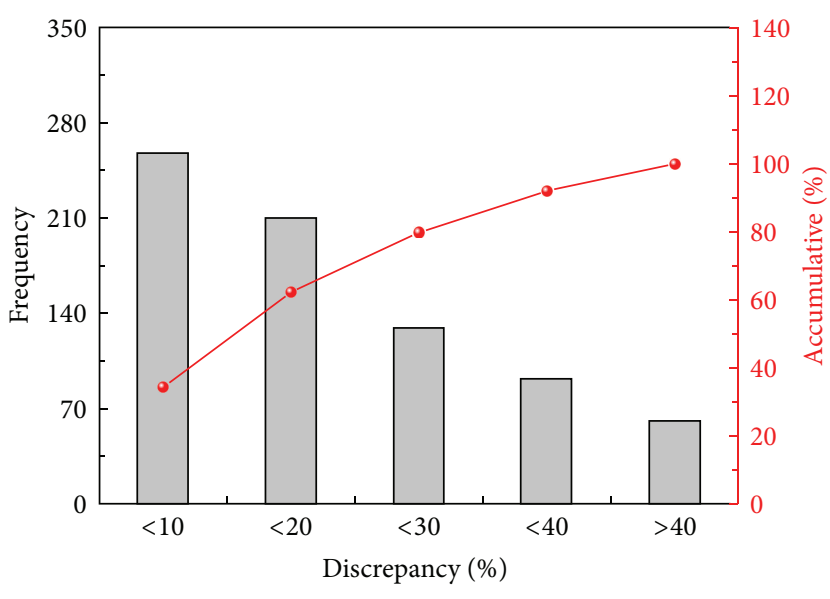

Beijing metro line 9

Discrepancy $(\%)=$ absolute value $[($ model value measured value)/measured value]

(a)

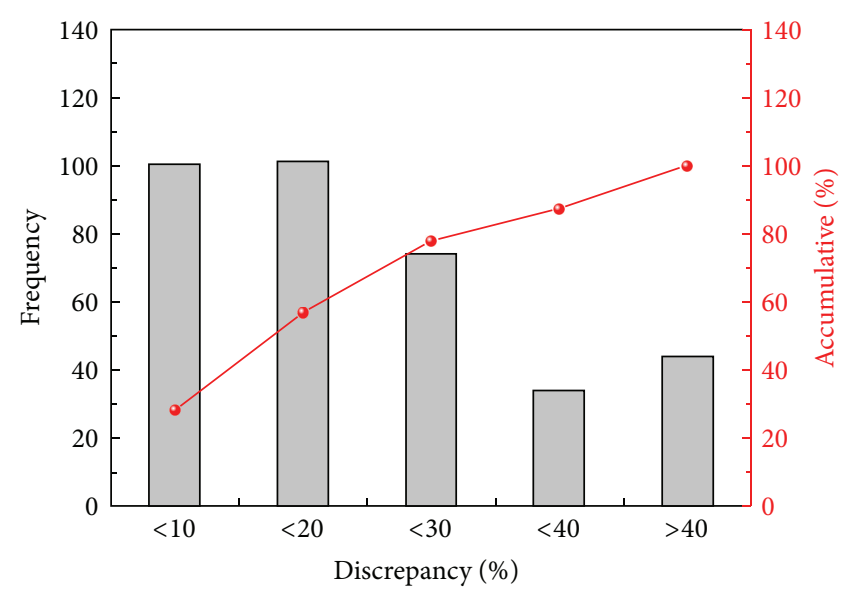

Shenzhen metro line 2 Discrepancy $(\%)=$ absolute value $[($ model value measured value)/measured value]

(b)

FIgURE 8: Statistical histogram of the model prediction errors. (a) Beijing Metro Line 9. (b) Shenzhen Metro Line 2.

TABLE 5: Engineering applications of the shield tunneling thrust model.

\begin{tabular}{|c|c|}
\hline $\begin{array}{l}\text { General formula of the } \\
\text { model }\end{array}$ & $F=\beta_{1} W \delta^{2}+\left(\beta_{2} W+\beta_{3} P\right) D^{2}$ \\
\hline $\begin{array}{l}\text { Thrust expression of } \\
\text { Beijing Metro Line } 9\end{array}$ & $F=9.1 \times 10^{3} W \delta^{2}+(0.61 W+0.39 P) 1$ \\
\hline $\begin{array}{l}\text { Thrust expression of } \\
\text { Shenzhen Metro Line } 2\end{array}$ & $F=8.9 \times 10^{3} W \delta^{2}+(0.59 W+0.39 P) D^{2}$ \\
\hline
\end{tabular}

\section{Conclusions}

A new method for the mass on-site data analysis and modeling of complex engineering equipment is proposed in this study, which integrates the mechanical dimension analysis and the regression analysis. First, the engineering variables were evaluated according to the mechanical equilibrium and the deformation compatibility; the primary and secondary influencing factors were then distinguished. Second, the internal interaction laws among different variables were determined through the dimensional analysis method. The basic architecture of the model was identified, and the general formula for the model was established. Third, the model coefficients were determined, and specific function expressions were provided through multiple regression analysis method in consideration of on-site data from specific projects. Lastly, the effectiveness and applicability of the model were verified through the residual analysis.

The method proposed was applied to analyze and model the tunneling thrust in shield engineering, determine the internal law of effect between the tunneling thrust and the core parameters (e.g., geological, equipment operating, and structural parameters), and build an explicit general tunneling thrust model. The model coefficients were identified, the thrust function expression of the specific project was provided, and the effectiveness of the model was verified based on the mass on-site data of Tianjin Metro Line 9. Furthermore, the model was also applied to two other engineering cases with different geological characteristics, Beijing Metro Line 9 and Shenzhen Metro Line 2, to evaluate the applicability of the model.

This work can serve as a theoretical basis for the thrust determination in the forward design and construction control of shield tunneling machines. It may also offer new research concepts for mass measured data analysis and modeling of complex engineering equipment characterized by nonlinearity, multiple influencing factors, and multifield coupling.

\section{Acknowledgments}

This work was supported by the State Key Laboratory of Shield Machine and Boring Technology, the National Basic Research Program of China (973 Program, Grant no. 2013CB035402), and the National Natural Science Foundation of China (NSFC, Grant no. 11127202).

\section{References}

[1] A. K. Choudhary, J. A. Harding, and M. K. Tiwari, "Data mining in manufacturing: a review based on the kind of knowledge," Journal of Intelligent Manufacturing, vol. 20, no. 5, pp. 501-521, 2009.

[2] S. Chatterjee and A. S. Hadi, Regression Analysis by Example, John Wiley \& Sons, Hoboken, NJ, USA, 2012.

[3] J. Zhao, P. Witold, and S. Sabrina, "Data mining and knowledge discovery in industrial engineering," Mathematical Problems in Engineering, vol. 2013, Article ID 790160, 2 pages, 2013.

[4] P. Z. Lu, S. Y. Chen, and Y. J. Zheng, "Artificial intelligence in civil engineering," Mathematical Problems in Engineering, vol. 2012, Article ID 145974, 22 pages, 2012. 
[5] W. Li, K. Huang, D. Zhang, and Z. Qin, "Accurate counting bloom filters for large-scale data processing," Mathematical Problems in Engineering, vol. 2013, Article ID 516298, 11 pages, 2013.

[6] L. T. Wang, G. F. Gong, H. Shi, and H. Y. Yang, "Modeling and analysis of thrust force for EPB shield tunneling machine," Automation in Construction, vol. 27, pp. 138-146, 2012.

[7] T. Krause, Schildvortrieb mit flüssigkeits- und erdgestützter Ortsbrust, vol. 24, Mitteilung des Instituts für Grundbau und Bodenmechanik, 1987, (German).

[8] B. Maidl, M. Herrenknecht, and L. Anheuser, Mechanised Shield Tunneling, Ernst \& Sohn, Berlin, Germany, 1996.

[9] H. Zhang, X. Wu, and W. Zeng, "Study on tunneling experiment and mathematical model of EPB shield," Chinese Journal of Rock Mechanics and Engineering, vol. 24, no. A02, pp. 5762-5766, 2005 (Chinese).

[10] U. Ates, N. Bilgin, and H. Copur, "Estimating torque, thrust and other design parameters of different type TBMs with some criticism to TBMs used in Turkish tunneling projects," Tunnelling and Underground Space Technology, vol. 40, pp. 4663, 2014.

[11] J. Hassanpour, J. Rostami, and J. Zhao, "A new hard rock TBM performance prediction model for project planning," Tunnelling and Underground Space Technology, vol. 26, no. 5, pp. 595-603, 2011.

[12] S. Yagiz, C. Gokceoglu, E. Sezer, and S. Iplikci, "Application of two non-linear prediction tools to the estimation of tunnel boring machine performance," Engineering Applications of Artificial Intelligence, vol. 22, no. 4-5, pp. 818-814, 2009.

[13] M. A. Grima, P. A. Bruines, and P. N. W. Verhoef, "Modeling tunnel boring machine performance by neuro-fuzzy methods," Tunnelling and Underground Space Technology, vol. 15, no. 3, pp. 259-269, 2000.

[14] Q. Zhang, C. Qu, Y. Kang, G. Huang, and Z. Cai, "Identification and optimization of energy consumption by shield tunnel machines using a combined mechanical and regression analysis," Tunnelling and Underground Space Technology, vol. 28, no. 1, pp. 350-354, 2012.

[15] Q. M. Gong and J. Zhao, "Influence of rock brittleness on TBM penetration rate in Singapore granite," Tunnelling and Underground Space Technology, vol. 22, no. 3, pp. 317-324, 2007.

[16] J. K. Hamidi, K. Shahriar, B. Rezai, and J. Rostami, "Performance prediction of hard rock TBM using Rock Mass Rating (RMR) system," Tunnelling and Underground Space Technology, vol. 25, no. 4, pp. 333-345, 2010.

[17] A. G. Benardos and D. C. Kaliampakos, "Modelling TBM performance with artificial neural networks," Tunnelling and Underground Space Technology, vol. 19, no. 6, pp. 597-605, 2004.

[18] C. Zhou, L. Y. Ding, and R. He, "PSO-based Elman neural network model for predictive control of air chamber pressure in slurry shield tunneling under Yangtze River," Automation in Construction, vol. 36, pp. 208-217, 2013.

[19] P. Rothman, "Forecasting asymmetric unemployment rates," Review of Economics and Statistics, vol. 80, no. 1, pp. 164-168, 1998.

[20] G. E. P. Box, G. M. Jenkins, and G. C. Reinsel, Time Series Analysis: Forecasting and Control, Holden-Day, San Francisco, Calif, USA, 1970. 


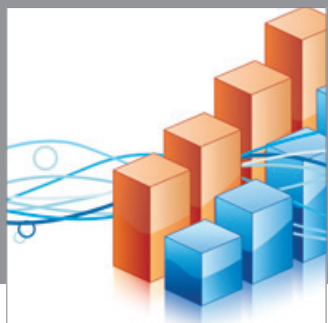

Advances in

Operations Research

mansans

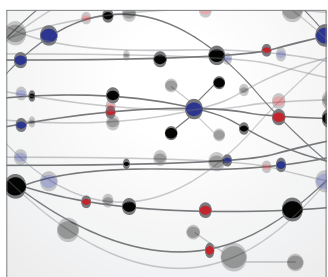

The Scientific World Journal
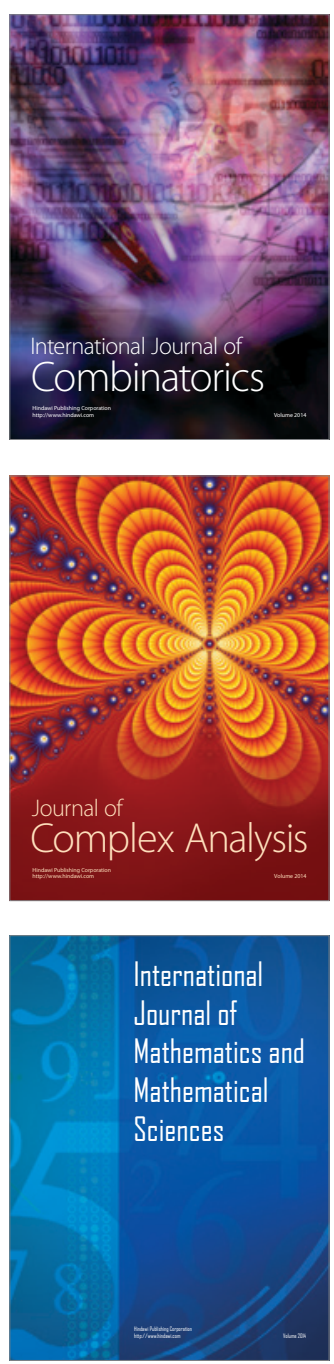
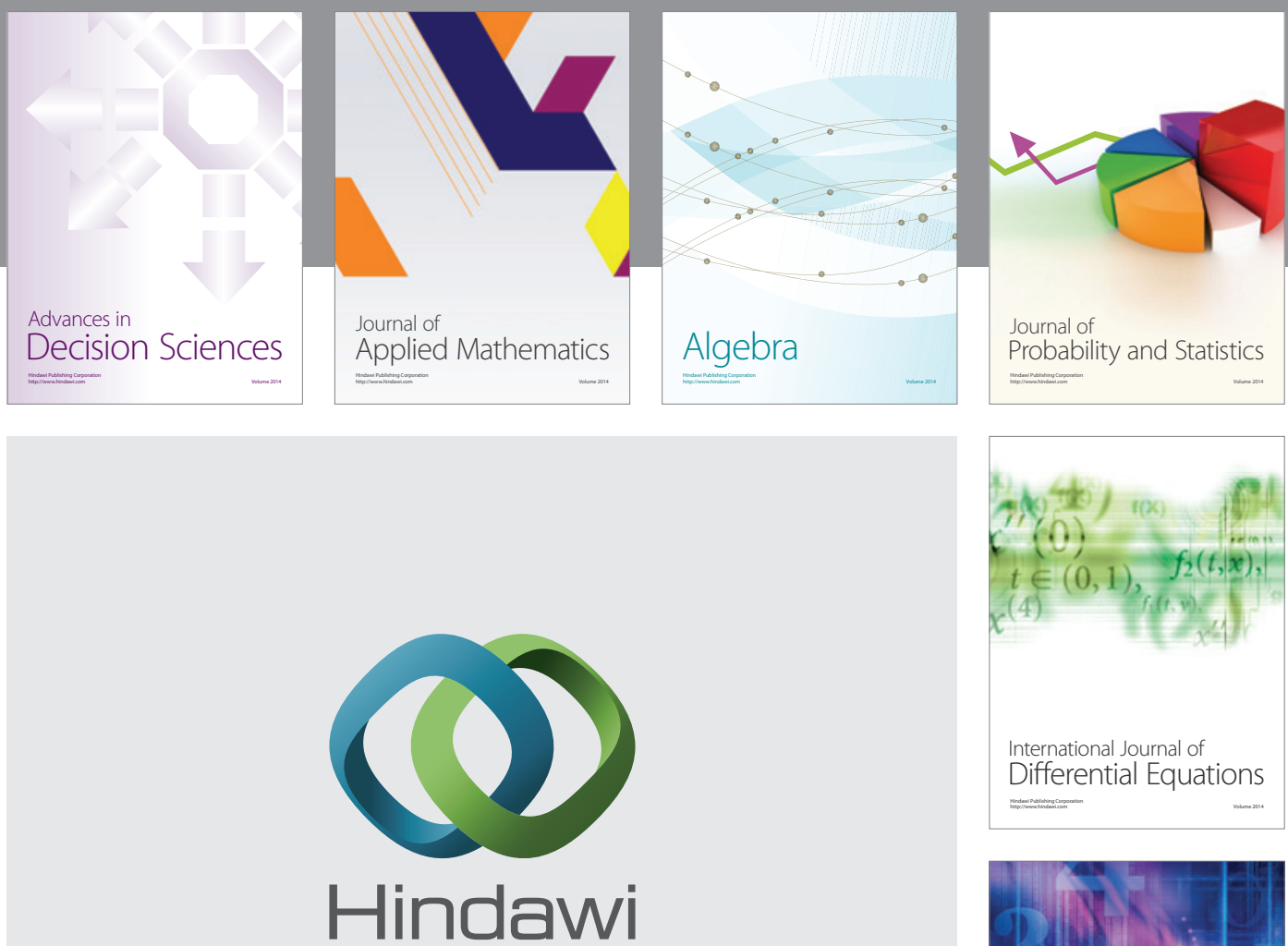

Submit your manuscripts at http://www.hindawi.com
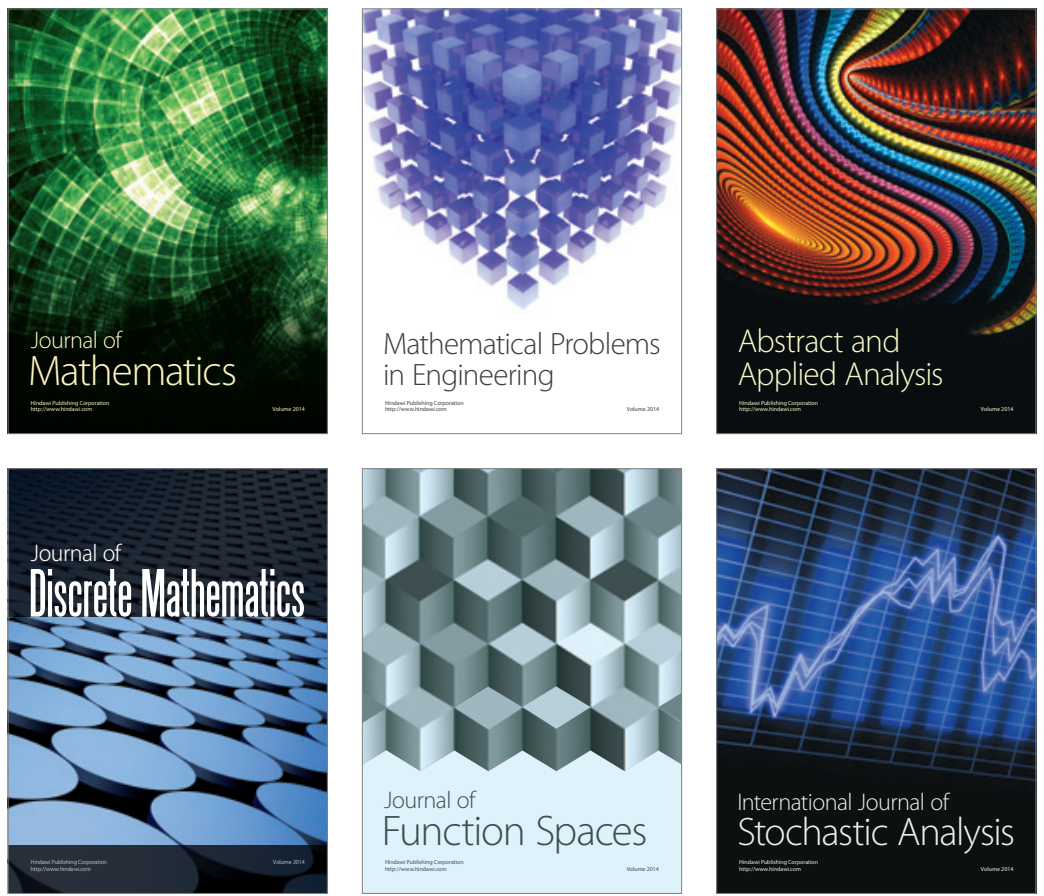

Journal of

Function Spaces

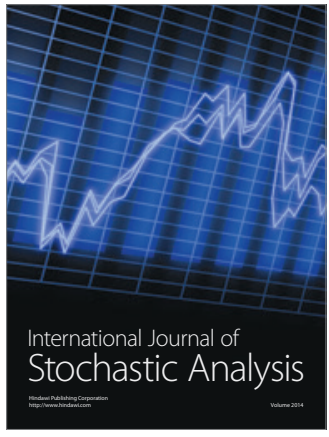

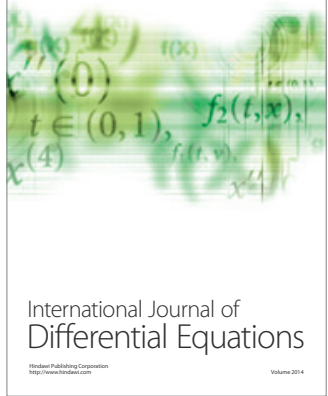
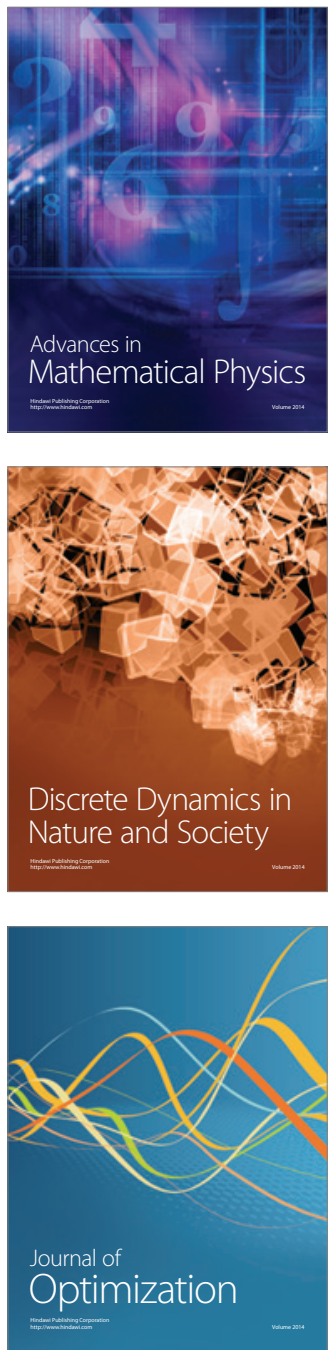